\title{
Acupuncture Modulates the Functional Connectivity of the Default Mode Network in Stroke Patients
}

\author{
Yong Zhang, ${ }^{1}$ Kuangshi Li, ${ }^{1}$ Yi Ren, ${ }^{1}$ Fangyuan Cui, ${ }^{1}$ Zijing Xie, ${ }^{1}$ Jae-Young Shin, ${ }^{1}$ \\ Zhongjian Tan, ${ }^{2}$ Lixin Tang, ${ }^{3}$ Lijun Bai, ${ }^{4}$ and Yihuai Zou ${ }^{1}$ \\ ${ }^{1}$ Department of Neurology and Stroke Center, Dongzhimen Hospital Affiliated to Beijing University of Chinese Medicine, \\ Beijing 100700, China \\ ${ }^{2}$ Department of Radiology, Dongzhimen Hospital Affiliated to Beijing University of Chinese Medicine, Beijing 100700, China \\ ${ }^{3}$ Department of Acupuncture, Dongzhimen Hospital Affiliated to Beijing University of Chinese Medicine, Beijing 100700, China \\ ${ }^{4}$ The Key Laboratory of Biomedical Information Engineering, Ministry of Education, Department of Biomedical Engineering, \\ School of Life Science and Technology, Xian Jiaotong University, Xian 710048, China
}

Correspondence should be addressed to Lijun Bai; bailj4152615@gmail.com and Yihuai Zou; zouyihuai2004@163.com

Received 28 December 2013; Revised 23 January 2014; Accepted 27 January 2014; Published 5 March 2014

Academic Editor: Baixiao Zhao

Copyright (C) 2014 Yong Zhang et al. This is an open access article distributed under the Creative Commons Attribution License, which permits unrestricted use, distribution, and reproduction in any medium, provided the original work is properly cited.

\begin{abstract}
Abundant evidence from previous fMRI studies on acupuncture has revealed significant modulatory effects at widespread brain regions. However, few reports on the modulation to the default mode network (DMN) of stroke patients have been investigated in the field of acupuncture. To study the modulatory effects of acupuncture on the DMN of stroke patients, eight right hemispheric infarction and stable ischemic stroke patients and ten healthy subjects were recruited to undergo resting state fMRI scanning before and after acupuncture stimulation. Functional connectivity analysis was applied with the bilateral posterior cingulate cortices chosen as the seed regions. The main finding demonstrated that the interregional interactions between the ACC and PCC especially enhanced after acupuncture at GB34 in stroke patients, compared with healthy controls. The results indicated that the possible mechanisms of the modulatory effects of acupuncture on the DMN of stroke patients could be interpreted in terms of cognitive ability and motor function recovery.
\end{abstract}

\section{Introduction}

The prevalence of stroke has increased in the past decades with the population aged and lifestyle changed [1]. Statistics from the American Heart Association state that there are more than 0.6 million people suffering from a new stroke in America every year [2]. As the leading cause of disability, stroke has greatly influenced the patients' quality of life and has left a huge burden and significant workload for their families and the whole nation [3].

Acupuncture, one of the most famous therapeutic modalities in traditional Chinese medicine, has emerged as an important modality of alternative and complementary therapeutic intervention in western medicine [4]. Recently, acupuncture has been widely used as a treatment for stroke based on a large body of clinical researches and systematic reviews which have drawn the preliminary conclusion that acupuncture is effective in stroke recovery [5-7]. However, the underlying mechanisms of these effects are not well understood.

In the last decades, noninvasive functional magnetic resonance imaging (fMRI) technique has opened a window into the brain, allowing us to investigate the central physiological function of acupuncture administration [8]. Converging evidence from fMRI studies has demonstrated that acupuncture stimulation can modulate neural activities in a wide cortico-subcortical network [9-11]. Neuroimaging studies of acupuncture have typically demonstrated extensive signal attenuations, mainly distributed in the medial temporal lobe, the posterior cingulate cortex (PCC), the medial prefrontal cortex (MPFC), and a large section of the parietal cortex [1214]. The spatial distribution of these deactivated regions has a prominent overlap with the core regions in a "default mode" network, which is mainly present at rest and whose activities 
TABLE 1: Demographic and clinical information about the stroke patients.

\begin{tabular}{|c|c|c|c|c|c|c|}
\hline Subject & Age (years) & Gender & Side & Lesion & MMSE & NIHSS \\
\hline 1 & 56 & $\mathrm{~F}$ & $\mathrm{R}$ & $B G$ & 22 & 14 \\
\hline 2 & 64 & M & $\mathrm{R}$ & IC & 30 & 3 \\
\hline 3 & 57 & $\mathrm{M}$ & $\mathrm{R}$ & IC & 27 & 9 \\
\hline 4 & 68 & $\mathrm{M}$ & $\mathrm{R}$ & CR & 29 & 5 \\
\hline 5 & 57 & $\mathrm{~F}$ & $\mathrm{R}$ & IC & 22 & 8 \\
\hline 6 & 58 & M & $\mathrm{R}$ & IC & 30 & 7 \\
\hline 7 & 71 & M & $\mathrm{R}$ & IC & 24 & 3 \\
\hline 8 & 52 & M & $\mathrm{R}$ & BG & 30 & 5 \\
\hline
\end{tabular}

F: female; M: male; R: right; L: left; BG: basal ganglia; IC: internal capsule; CR: corona radiate; NIHSS: National Institute of Health Stroke Scale; MMSE: MiniMental State Examination.

are strongly reduced during various goal-directed tasks [15, 16]. Similarly, acupuncture, not the sham condition, can also induce the increased connectivity within both the default mode network (DMN) and the sensorimotor network (SMN) [17]. It has also been reported that acupuncture at a certain acupoint, like Neiguan (PC6) and Guangming (GB37), could alter the intrinsic functional connectivity in the DMN of healthy subjects $[17,18]$.

Previous fMRI studies have reported specific functional connectivity changes in the DMN of stroke patients. Decreased functional connectivity in the PCC, the medial temporal lobe, and the medial prefrontal cortical areas within the DMN and reduced interregional functional connectivity between these regions have been found in stroke patients [19]. The abnormalities of the DMN functional connectivity might underlie the loss of episodic memory [19] and the occurrence of post-stroke depression and anxiety [20]. Therefore, understanding of DMN dysfunctions of stroke patients may help us comprehend the pathological mechanisms of stroke and provide additional information on brain function induced by rehabilitative interventions [21].

Given that acupuncture is effective in stroke rehabilitation and could alter the DMN in healthy subjects, we hypothesized that acupuncture would also modulate the DMN in stroke patients and this would give more interpretation of the effect of acupuncture. With healthy subjects as controls, in the current study, we tried to investigate the changes of restingstate functional connectivity in the DMN of stroke patients evoked by acupuncture at Yanglingquan (GB34), a frequently used acupoint to treat hemiplegia.

\section{Materials and Methods}

2.1. Subjects. A total of 8 stable ischemic stroke patients (6 males, mean age: $60.4 \pm 6.6$ years), diagnosed with right hemispheric corona radiate, internal capsule or basal ganglia infarction by MRI with unilateral limb disability, were recruited from Dongzhimen Hospital Affiliated to Beijing University of Chinese Medicine. All 8 patients met the following inclusion criteria: (1) first episode of stroke; (2) being between 35 and 80 years old; (3) being righthanded; (4) 2-12 weeks after the onset of stroke; (5) sufficient cognition to follow simple commands, MMSE (Mini-Mental State Examination score) $>21$. Patients were excluded if they met any of the following criteria: (1) bilateral infarcts; (2) recurrent stroke; (3) any previous history of alcohol or drug abuse; (4) history of epilepsy or other neurological diseases and psychiatric disorders; (5) serious cognitive deficits; (6) being with any MRI contraindications. Table 1 summarizes the demographic and clinical information about the stroke patients.

Another 10 right-handed normal subjects ( 7 males, mean age: $59.1 \pm 7.8$ years) were recruited to serve as healthy controls. All 10 healthy controls passed normal neurological examination and had no history of epilepsy or other neurological diseases, psychiatric disorders, or any MRI contraindications. Written informed consent was obtained from all subjects. The data was analyzed anonymously. All research procedures were approved by the ethical committee of Dongzhimen Hospital Affiliated to Beijing University of Chinese Medicine and conducted in accordance with the Declaration of Helsinki.

2.2. Experimental Paradigm. A multiblock paradigm is generally used in fMRI studies, which implicitly presumes the temporal intensity profiles of the certain event conforming to the "on-off" specifications. Since the acupuncture action is slow to develop and resolve [22], the temporal aspects of the BOLD response to acupuncture may violate the assumptions of the block-designed estimates. In addition, using several stimulation blocks in a short period of time, investigators may not be able to dissociate the long-lasting effects from other confounding changes, such as the effect of needle manipulation during the experiment $[9,23]$. In the current study, we adopted a new experimental paradigm, namely, the nonrepeated event-related-fMRI (NRER-fMRI) design [16, 24], to investigate such prolonged effects after acupuncture administration.

The NRER-fMRI design paradigm was employed during the acupuncture stimulation, incorporating 1-minute needle manipulation, preceded by 1-minute resting epoch and followed by 8 -minute resting scan (without acupuncture manipulation). Acupuncture was performed at Yanglingquan (GB34, located in the lateral aspect of the posterior knee 
[25]) on the left leg. The acupoint GB34, regarded as the meeting point of sinews and tendons according to the theory of Chinese medicine, is often used in the treatment of hemiplegia and motor dysfunction after stroke. Acupuncture was performed by inserting a sterile, single-use silver needle (25 $\mathrm{mm}$ in length and $0.30 \mathrm{~mm}$ in diameter) vertically into GB34 to a depth of $2-3 \mathrm{~cm}$, and the stimulation consisted of rotating the needle clockwise and counterclockwise at $1 \mathrm{~Hz}$ with even reinforcing and reducing manipulation for 60 s. All acupuncture procedures were performed by the same experienced and licensed acupuncturist. Another 8minute resting scan was performed before the acupuncture procedure as the baseline.

De-qi is believed to be essential to the therapeutic effectiveness of acupuncture and is often used as a signal to acupuncturists that the proper amount of needle stimulation is being performed [26, 27]. At the end of each fMRI scanning, subjects were asked to complete questionnaires to rate their experience of De-qi. Because sharp pain was considered as an inadvertent noxious stimulation, we excluded the subjects from further analysis if they experienced the sharp pain. Among all the participants, none experienced the sharp pain. Related results have been described in another paper [28].

2.3. Data Acquisition. fMRI images were acquired using a 3.0T MRI scanner (Siemens, Sonata, Germany). During scanning, subjects remained in the supine position with their heads immobilized by a custom-built head holder to prevent head movements. Subjects wore earplugs throughout the experiment to attenuate MRI gradient noise. Thirty-two axial slices $(\mathrm{FOV}=225 \mathrm{~mm} \times 225 \mathrm{~mm}$, matrix $=64 \times 64$, and thickness $=3.5 \mathrm{~mm}$ ) parallel to the AC-PC plane and covering the whole brain were obtained using a T2-weighted singleshot, gradient-recalled echo planar imaging (EPI) sequence $\left(\mathrm{TR}=2000 \mathrm{~ms}, \mathrm{TE}=30 \mathrm{~ms}\right.$, and flip angle $\left.=90^{\circ}\right)$. Prior to the functional run, high-resolution structural information on each subject was also acquired using 3D MRI sequences with a voxel size of $1 \mathrm{~mm}^{3}$ for anatomical localization $(\mathrm{TR}=$ $1900 \mathrm{~ms}, \mathrm{TE}=2.52 \mathrm{~ms}$, flip angle $=90^{\circ}$, matrix $=256 \times 256$, FOV $=250 \mathrm{~mm} \times 250 \mathrm{~mm}$, and slice thickness $=1 \mathrm{~mm}$ ).

2.4. Definition of Region of Interest. The region of interest (ROI) was located in the bilateral posterior cingulate cortex (PCC, centered Montreal Neurological Institute coordinates: $0,-56$, and 25 ), which was widely reported to be connected with other brain regions of the DMN [29] and associated with the sustained modulation effects of acupuncture [18].

2.5. fMRI Data Analysis. The data included the baseline resting scan and the post-acupuncture resting scan. For each resting scan, the first 10 time points were discarded to avoid the instability of the initial MRI signal. All preprocessing steps were carried out using statistical parametric mapping (SPM5, http://www.fil.ion.ucl.ac.uk/spm/). The images were first time-sliced and then realigned to correct for head motions. The image data was further processed with spatial normalization based on the MNI space and resampled at $3 \mathrm{~mm} \times 3 \mathrm{~mm} \times 3 \mathrm{~mm}$. The functional images were spatially smoothed with an $8 \mathrm{~mm}$ full-width-at-halfmaximum (FWHM) Gaussian kernel and then processed with a bandpass filter of $0.01-0.10 \mathrm{~Hz}$. For the calculation of the functional connectivity, the correlation coefficient of each voxel was the average of the correlations (or anticorrelations) with ROI and was later normalized to $Z$-scores with Fisher's r-to- $z$ transformation to acquire the entire brain $Z$-score map of each subject. For the group-level analyses, the functional connectivity was conducted by two-sample $t$-test and paired $t$-test using SPM5 software. The reported statistics were colorcoded and mapped in Talairach space.

\section{Results}

In the resting state, the PCC showed attenuated functional connectivity with the following brain regions such as the right inferior parietal lobules, bilateral superior frontal gyrus, right middle frontal gyrus, right medial frontal gyrus, right middle temporal gyrus, right inferior temporal gyrus, and right fusiform gyrus in stroke patients compared with healthy controls. By contrast, the PCC showed increased functional connectivity with the following regions such as the bilateral precentral gyrus, bilateral postcentral gyrus, right inferior frontal gyrus, bilateral inferior parietal lobules, bilateral superior temporal gyrus, and bilateral insula in stroke patients compared with the healthy controls. Specific cluster locations are shown in Table 2.

During the post-acupuncture resting state, the PCC showed significantly increased functional connectivity with the left middle temporal gyrus and superior temporal gyrus in healthy controls, in comparison with baseline state. For the stroke patients, acupuncture can particularly enhance the functional connectivity between the PCC and bilateral anterior cingulate cortex (ACC), while decreased functional connectivity was primarily located in the left postcentral gyrus and precentral gyrus (shown in Figure 1). Specific cluster locations are shown in Table 3.

\section{Discussion}

In the present study, resting-state fMRI scanning was applied before and after acupuncture stimulation on both stroke patients and control subjects. Resting-state functional connectivity of the DMN was analyzed, respectively, with the PCC chosen as the seed region. By doing this, we tried to test the hypothesis that acupuncture could modulate the DMN in stroke patients. The results could be divided into three continuous parts. Firstly, we detected decreased functional connectivity in the DMN of stroke patients, which is compatible to previous studies. Secondly, our results provided supportive evidence that acupuncture could modulate the DMN in healthy subjects. Finally, increased functional connectivity between the PCC and ACC was observed in the DMN of stroke patients after acupuncture at GB34, which might serve as a possible interpretation of the modulatory effects of acupuncture. 
TABLE 2: Results of the control subjects and stroke patients before acupuncture.

\begin{tabular}{|c|c|c|c|c|c|c|}
\hline \multirow{2}{*}{ Regions } & \multirow{2}{*}{ Side } & \multicolumn{3}{|c|}{ Talairach } & \multirow{2}{*}{$t$-value } & \multirow{2}{*}{ Voxels } \\
\hline & & $X$ & $Y$ & $Z$ & & \\
\hline \multicolumn{7}{|c|}{ Decreased connectivity to the PCC in stroke patients } \\
\hline Inferior parietal lobule & $\mathrm{R} / \mathrm{IL}$ & 45 & -62 & 47 & 4.17 & 37 \\
\hline Superior frontal gyrus & $\mathrm{L} / \mathrm{CL}$ & -6 & 20 & 60 & 4.32 & 117 \\
\hline Superior frontal gyrus & $\mathrm{R} / \mathrm{IL}$ & 3 & 40 & 45 & 4.55 & 193 \\
\hline Middle frontal gyrus & $\mathrm{R} / \mathrm{IL}$ & 24 & 37 & 42 & 4.16 & 33 \\
\hline Medial frontal gyrus & $\mathrm{R} / \mathrm{IL}$ & 6 & 58 & 3 & 4.10 & 80 \\
\hline Middle temporal gyrus & $\mathrm{R} / \mathrm{IL}$ & 53 & -66 & 28 & 4.22 & 28 \\
\hline Fusiform gyrus & $\mathrm{R} / \mathrm{IL}$ & 59 & -10 & -22 & 5.28 & 30 \\
\hline \multicolumn{7}{|c|}{ Increased connectivity to the PCC in stroke patients } \\
\hline Precentral gyrus & $\mathrm{R} / \mathrm{IL}$ & 62 & 6 & 11 & 5.10 & 55 \\
\hline Precentral gyrus & $\mathrm{L} / \mathrm{CL}$ & -48 & -2 & 8 & 4.60 & 235 \\
\hline Postcentral gyrus & $\mathrm{R} / \mathrm{IL}$ & 62 & -19 & 31 & 5.49 & 152 \\
\hline Postcentral gyrus & $\mathrm{L} / \mathrm{CL}$ & -62 & -5 & 17 & 4.78 & 162 \\
\hline Inferior parietal lobule & $\mathrm{R} / \mathrm{IL}$ & 62 & -22 & 29 & 4.84 & 163 \\
\hline Inferior parietal lobule & $\mathrm{L} / \mathrm{CL}$ & -53 & -28 & 21 & 4.52 & 136 \\
\hline Inferior frontal gyrus & $\mathrm{R} / \mathrm{IL}$ & 50 & 5 & 33 & 7.48 & 62 \\
\hline Superior temporal gyrus & $\mathrm{R} / \mathrm{IL}$ & 50 & 14 & -8 & 4.82 & 39 \\
\hline Superior temporal gyrus & $\mathrm{L} / \mathrm{CL}$ & -56 & -25 & 15 & 4.49 & 65 \\
\hline Insula & $\mathrm{R} / \mathrm{IL}$ & 36 & -11 & 9 & 4.97 & 111 \\
\hline Insula & $\mathrm{L} / \mathrm{CL}$ & -33 & -14 & 15 & 4.86 & 171 \\
\hline
\end{tabular}

Results from two-sample $t$-test $(\mathrm{df}=16, P<0.01$, uncorrected, corrected by Monte Carlo Simulations, iterated 1000 times, and cluster size $>76$ voxels). R: right; L: left; IL: ipsilateral; CL: contralateral.

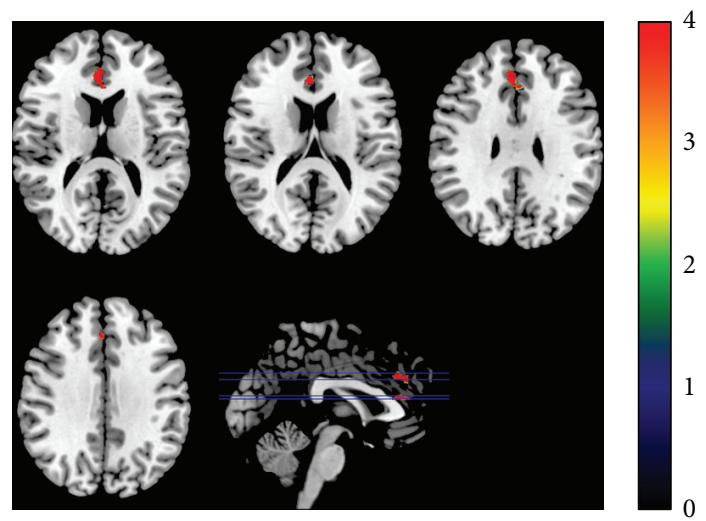

FIGURE 1: Brain regions showed increased connectivity to the PCC in stroke patients after acupuncture. Increased functional connectivity to the PCC was observed in the bilateral ACC after acupuncture in stroke patients. Results from two-sample $t$-test $(\mathrm{df}=16, P<0.01$, uncorrected, corrected by Monte Carlo Simulations, and iterated 1000 times).

4.1. Resting-State Functional Connectivity before Acupuncture. We compared the resting-state functional connectivity anchored by PCC between the control subjects and stroke patients. There were a set of brain regions showing decreased or increased functional connectivity in stroke patients in comparison with the control subjects. Most of the decreased regions were involved in the frontal lobe and temporal lobe, which partly overlapped with the medial temporal lobe (MTL) and the medial prefrontal cortical (MPFC) areas within the main regions of the DMN. These results were consistent with a recent study that detected decreased functional connectivity in the PCC, MTL, and MPFC [19]. All stroke patients involved in our study were diagnosed with right hemispheric infraction. Based on our findings, most of the brain regions showed decreased functional connectivity to the PCC located in the right hemisphere which was consistent with the infraction lesions. This has confirmed the accuracy of our results to some extent. At the same time, some brain regions which were closely related to the motor and sensory functions showed increased functional connectivity to the PCC. These increases could be interpreted as compensatory reallocation or recruitment of motor and sensory functions 
TABLE 3: Results of the control subjects and stroke patients after acupuncture procedures.

\begin{tabular}{|c|c|c|c|c|c|c|}
\hline \multirow{2}{*}{ Regions } & \multirow{2}{*}{ Side } & \multicolumn{3}{|c|}{ Talairach } & \multirow{2}{*}{$t$-value } & \multirow{2}{*}{ Voxels } \\
\hline & & $X$ & $Y$ & $Z$ & & \\
\hline \multicolumn{7}{|c|}{ Increased connectivity to the PCC in control subjects } \\
\hline Middle temporal gyrus & $\mathrm{L} / \mathrm{CL}$ & -42 & -61 & 9 & 5.84 & 34 \\
\hline Superior temporal gyrus & $\mathrm{L} / \mathrm{CL}$ & -48 & -46 & 19 & 6.07 & 29 \\
\hline \multicolumn{7}{|c|}{ Increased connectivity to the PCC in stroke patients } \\
\hline Anterior cingulate & $\mathrm{L} / \mathrm{CL}$ & -6 & 30 & 23 & 6.45 & 21 \\
\hline Anterior cingulate & $\mathrm{R} / \mathrm{IL}$ & 3 & 33 & 12 & 5.63 & 28 \\
\hline \multicolumn{7}{|c|}{ Decreased connectivity to the PCC in stroke patients } \\
\hline Precentral gyrus & $\mathrm{L} / \mathrm{CL}$ & -62 & 1 & 19 & 6.47 & 70 \\
\hline Postcentral gyrus & $\mathrm{L} / \mathrm{CL}$ & -59 & -17 & 15 & 5.39 & 41 \\
\hline
\end{tabular}

Results from two-sample $t$-test ( $\mathrm{df}=16, P<0.01$, uncorrected, corrected by Monte Carlo Simulations, iterated 1000 times, and cluster size $>76$ voxels). R: right; L: left; IL: ipsilateral; CL: contralateral.

during the functional reorganization procedures of brain networks as have been confirmed by previous fMRI studies [30-32].

\subsection{Acupuncture Modulates the DMN in Healthy Subjects. In} the current resting-state fMRI study, we observed increased functional connectivity to the PCC in the left middle temporal gyrus and the left superior temporal gyrus after acupuncture at GB34 compared with that before acupuncture in healthy subjects. Another resting-state fMRI study showed that acupuncture at Neiguan (PC6) induced increased DMN connectivity with pain, affective and memory regions including the ACC, the hippocampal formation, and the middle temporal gyrus [17]. Other resting-state fMRI studies have demonstrated that both the PCC and the anterior insula play key roles in the modulatory effects of different acupoints on the DMN in healthy subjects $[16,18,33]$. A task related fMRI study revealed that the brain regions both deactivated and activated by acupuncture at different acupoints overlapped with the DMN in healthy subjects [34]. Previous fMRI studies have drawn the conclusion that acupuncture could modulate the DMN in healthy subjects [35]. However, different modulation patterns exist among different acupoints due to the acupoint specificity $[18,34,35]$, which suggested that the regions involved in fMRI studies with different acupoints may differ from each other.

4.3. Acupuncture Modulates the DMN of Stroke Patients. Several studies focusing on the neural effects of acupuncture on stroke patients have already been carried out [36-38]. However, there are few published studies available examining the effects of acupuncture on the DMN in stroke patients. In the current study, we applied resting-state functional connectivity to investigate the modulatory effects of acupuncture on the DMN of stroke patients with healthy subjects as control. We found that the ACC which overlap with regions underlying the DMN showed significantly increased functional connectivity with the PCC in stroke patients after acupuncture at GB34.

The ACC and PCC are considered as important components of the DMN [29]. Both PCC and ACC are regarded as core regions involved in memory and cognitive processing and their interactions with other brain networks may be important for conscious awareness $[39,40]$. The DMN is an intrinsic resting-state network that regulates self-referential activity and consciousness [29] and its functional connectivity is decreased in stroke and other neurodegenerative diseases [19]. Thus, we inferred that the modulatory effects of acupuncture on the DMN of stroke patients could partly be interpreted as the recovery of cognitive ability.

A previous study focusing on the transition from rest to movement proved that the PCC and ACC served as important interaction hubs of the DMN and sensorimotor network (SMN) during movement-readiness state [41] indicating that the PCC and ACC are the overlapped key regions of the DMN and SMN. The structural and functional reorganization of the SMN after stroke has been confirmed by previous studies [42]. Previous task related fMRI studies focusing on the neurological effects of acupuncture at GB34 also induced a set of motor related brain regions involved in the SMN [43, 44]. Thus, the increased functional connectivity between the PCC and ACC induced by acupuncture at GB34 in our study could also be interpreted as the effects of the enhancement of motor recovery. We also observed decreased functional connectivity in the left precentral gyrus and the left postcentral gyrus which were underlying the brain regions of the SMN. In the present study, we propose that further studies focusing on the modulatory effects of acupuncture on the SMN, an anticorrelated network of the DMN, are still needed to give comprehensive explanations of the effects of acupuncture.

4.4. Limitations of This Study. However, we have to point out that the results represented here were just a preliminary exploration to the effects of acupuncture on the DMN of stroke patients. Further studies with larger sample size are still needed to confirm these results.

\section{Conclusions}

In summary, the main finding demonstrated that acupuncture at GB34 in stroke patients mainly induced enhanced functional connectivity between the ACC and PCC, in 
comparison with the healthy controls. We inferred that the modulatory effects of acupuncture on the DMN in stroke patients could be interpreted in terms of cognitive ability and motor function recovery. Further studies with larger sample size and focusing on the effects of acupuncture on the connection between the DMN and SMN are also needed.

\section{Conflict of Interests}

The authors declare that there is no conflict of interests regarding the publication of this paper.

\section{Authors' Contribution}

Yong Zhang and Kuangshi Li contributed equally to this work.

\section{Acknowledgments}

This paper is supported by the National Natural Science Foundation of China (Grants nos. 81072773, 81071217, 81371630, and 30672690), the Beijing Nova Program (Grant no. Z111101054511116), the Beijing Natural Science Foundation (Grant no. 4122082), the Beijing Young Talent Program of Beijing Education Committee, the Fundamental Research Funds for the Central Universities, and the Middle-Aged Teachers Research Funds of Beijing University of Chinese Medicine (Grant no. 2013JYBZZJS148).

\section{References}

[1] M. Liu, B. Wu, W.-Z. Wang, L.-M. Lee, S.-H. Zhang, and L.-Z. Kong, "Stroke in China: epidemiology, prevention, and management strategies," The Lancet Neurology, vol. 6, no. 5, pp. 456464, 2007.

[2] V. L. Roger, A. S. Go, D. M. Lloyd-Jones et al., "Executive summary: heart disease and stroke statistics-2012 update: a report from the American heart association," Circulation, vol. 125, no. 1, pp. 188-197, 2012.

[3] G. A. Donnan, M. Fisher, M. Macleod, and S. M. Davis, "Stroke," The Lancet, vol. 371, no. 9624, pp. 1612-1623, 2008.

[4] "NIH consensus conference: acupuncture," Journal of the American Medical Association, vol. 280, no. 17, pp. 1518-1524, 1998.

[5] J. C. Kong, M. S. Lee, B.-C. Shin, Y.-S. Song, and E. Ernst, "Acupuncture for functional recovery after stroke: a systematic review of sham-controlled randomized clinical trials," Canadian Medical Association Journal, vol. 182, no. 16, pp. 1723-1729, 2010.

[6] P. Wu, E. Mills, D. Moher, and D. Seely, "Acupuncture in poststroke rehabilitation: a systematic review and meta-analysis of randomized trials," Stroke, vol. 41, no. 4, pp. el71-e179, 2010.

[7] Y. Zhang, H. Jin, D. Ma et al., "Efficacy of integrated rehabilitation techniques of traditional chinese medicine for ischemic stroke: a randomized controlled trial," The American Journal of Chinese Medicine, vol. 41, no. 05, pp. 971-981, 2013.

[8] L. Bai and L. Lao, "Neurobiological foundations of acupuncture: the relevance and future prospect based on neuroimaging evidence," Evidence-Based Complementary and Alternative Medicine, vol. 2013, Article ID e812568, 9 pages, 2013.
[9] L. Bai, W. Qin, J. Tian et al., "Time-varied characteristics of acupuncture effects in fMRI studies," Human Brain Mapping, vol. 30, no. 11, pp. 3445-3460, 2009.

[10] L. Bai, J. Tian, C. Zhong et al., "Acupuncture modulates temporal neural responses in wide brain networks: evidence from fMRI study," Molecular Pain, vol. 6, article 73, 2010.

[11] W. Huang, D. Pach, V. Napadow et al., "Characterizing acupuncture stimuli using brain imaging with fMRI: a systematic review and meta-analysis of the literature," PLoS ONE, vol. 7, no. 4, Article ID e32960, 2012.

[12] S.-S. Yoo, E.-K. Teh, R. A. Blinder, and F. A. Jolesz, "Modulation of cerebellar activities by acupuncture stimulation: evidence from fMRI study," NeuroImage, vol. 22, no. 2, pp. 932-940, 2004.

[13] K. K. S. Hui, J. Liu, O. Marina et al., "The integrated response of the human cerebro-cerebellar and limbic systems to acupuncture stimulation at ST 36 as evidenced by fMRI," NeuroImage, vol. 27, no. 3, pp. 479-496, 2005.

[14] M.-T. Wu, J.-C. Hsieh, J. Xiong et al., "Central nervous pathway for acupunture stimulation: localization of processing with functional MR imaging of the brain: preliminary experience," Radiology, vol. 212, no. 1, pp. 133-141, 1999.

[15] M. E. Raichle, A. M. MacLeod, A. Z. Snyder, W. J. Powers, D. A. Gusnard, and G. L. Shulman, "A default mode of brain function," Proceedings of the National Academy of Sciences of the United States of America, vol. 98, no. 2, pp. 676-682, 2001.

[16] L. Bai, W. Qin, J. Tian et al., "Acupuncture modulates spontaneous activities in the anticorrelated resting brain networks," Brain Research, vol. 1279, pp. 37-49, 2009.

[17] R. P. Dhond, C. Yeh, K. Park, N. Kettner, and V. Napadow, "Acupuncture modulates resting state connectivity in default and sensorimotor brain networks," Pain, vol. 136, no. 3, pp. 407418, 2008.

[18] P. Liu, Y. Zhang, G. Zhou et al., "Partial correlation investigation on the default mode network involved in acupuncture: an fMRI study," Neuroscience Letters, vol. 462, no. 3, pp. 183-187, 2009.

[19] A. M. Tuladhar, L. Snaphaan, E. Shumskaya et al., "Default mode network connectivity in stroke patients," PLOS ONE, vol. 8, no. 6, Article ID e66556, 2013.

[20] S. Lassalle-Lagadec, I. Sibon, B. Dilharreguy, P. Renou, O. Fleury, and M. Allard, "Subacute default mode network dysfunction in the prediction of post-stroke depression severity," Radiology, vol. 264, no. 1, pp. 218-224, 2012.

[21] S. Whitfield-Gabrieli and J. M. Ford, "Default mode network activity and connectivity in psychopathology," Annual Review of Clinical Psychology, vol. 8, no. 6, pp. 49-76, 2012.

[22] D. J. Mayer, "Acupuncture: an evidence-based review of the clinical literature," Annual Review of Medicine, vol. 51, pp. 4963, 2000.

[23] Y. Zhang, W. Qin, P. Liu et al., "An fMRI study of acupuncture using independent component analysis," Neuroscience Letters, vol. 449, no. 1, pp. 6-9, 2009.

[24] W. Qin, J. Tian, L. Bai et al., "FMRI connectivity analysis of acupuncture effects on an amygdala-associated brain network," Molecular Pain, vol. 4, article 55, pp. 1-17, 2008.

[25] W. H. Organization, WHO Standard Acupuncture Point Locations in the Western Pacific Region, WHO Western Pacific Region, Geneva, Switzerland, 2008.

[26] J. Park, H. Park, H. Lee, S. Lim, K. Ahn, and H. Lee, "Deqi sensation between the acupuncture-experienced and the Naïve: a Korean study II," The American Journal of Chinese Medicine, vol. 33, no. 2, pp. 329-337, 2005. 
[27] C. Witt, B. Brinkhaus, S. Jena et al., "Acupuncture in patients with osteoarthritis of the knee: a randomised trial," The Lancet, vol. 366, no. 9480, pp. 136-143, 2005.

[28] L. Bai, F. Cui, Y. Zou, and L. Lao, "Acupuncture De Qi in stable somatosensory stroke patients: relations with effective brain network for motor recovery," Evidence-Based Complementary and Alternative Medicine, vol. 2013, Article ID e197238, 9 pages, 2013.

[29] M. D. Greicius, B. Krasnow, A. L. Reiss, and V. Menon, "Functional connectivity in the resting brain: a network analysis of the default mode hypothesis," Proceedings of the National Academy of Sciences of the United States of America, vol. 100, no. 1, pp. 253-258, 2003.

[30] C. Grefkes and G. R. Fink, "Reorganization of cerebral networks after stroke: new insights from neuroimaging with connectivity approaches," Brain, vol. 134, no. 5, pp. 1264-1276, 2011.

[31] L. Wang, C. Yu, H. Chen et al., "Dynamic functional reorganization of the motor execution network after stroke," Brain, vol. 133, no. 4, pp. 1224-1238, 2010.

[32] C. Calautti and J.-C. Baron, "Functional neuroimaging studies of motor recovery after stroke in adults: a review," Stroke, vol. 34, no. 6, pp. 1553-1566, 2003.

[33] Y. You, L. Bai, R. Dai et al., "Altered Hub configurations within default mode network following acupuncture at ST36: a multimodal investigation combining fMRI and MEG," PLoS ONE, vol. 8, no. 5, Article ID e64509, 2013.

[34] K. K. S. Hui, O. Marina, J. D. Claunch et al., "Acupuncture mobilizes the brain's default mode and its anti-correlated network in healthy subjects," Brain Research, vol. 1287, pp. 84103, 2009.

[35] K. K. S. Hui, O. Marina, J. Liu, B. R. Rosen, and K. K. Kwong, "Acupuncture, the limbic system, and the anticorrelated networks of the brain," Autonomic Neuroscience, vol. 157, no. 1-2, pp. 81-90, 2010.

[36] G. Li, C. R. Jack Jr., and E. S. Yang, "An fMRI study of somatosensory-implicated acupuncture points in stable somatosensory stroke patients," Journal of Magnetic Resonance Imaging, vol. 24, no. 5, pp. 1018-1024, 2006.

[37] J. D. Lee, J. S. Chon, H. K. Jeong et al., "The cerebrovascular response to traditional acupuncture after stroke," Neuroradiology, vol. 45, no. 11, pp. 780-784, 2003.

[38] J. D. Schaechter, B. D. Connell, W. B. Stason et al., "Correlated change in upper limb function and motor cortex activation after verum and sham acupuncture in patients with chronic stroke," Journal of Alternative and Complementary Medicine, vol. 13, no. 5, pp. 527-532, 2007.

[39] G. Bush, P. Luu, and M. I. Posner, "Cognitive and emotional influences in anterior cingulate cortex," Trends in Cognitive Sciences, vol. 4, no. 6, pp. 215-222, 2000.

[40] C. Lavin, C. Melis, E. Mikulan, C. Gelormini, D. Huepe, and A. Ibanez, "The anterior cingulate cortex: an integrative hub for human socially-driven interactions," Frontiers in Neuroscience, vol. 7, no. 64, pp. 1-4, 2013.

[41] S. Treserras, K. Boulanouar, F. Conchou et al., "Transition from rest to movement: brain correlates revealed by functional connectivity," NeuroImage, vol. 48, no. 1, pp. 207-216, 2009.

[42] M. P. A. van Meer, K. van der Marel, K. Wang et al., "Recovery of sensorimotor function after experimental stroke correlates with restoration of resting-state interhemispheric functional connectivity," Journal of Neuroscience, vol. 30, no. 11, pp. 39643972, 2010.
[43] B.-J. Na, G.-H. Jahng, S.-U. Park et al., "An fMRI study of neuronal specificity of an acupoint: electroacupuncture stimulation of Yanglingquan (GB34) and its sham point," Neuroscience Letters, vol. 464, no. 1, pp. 1-5, 2009.

[44] S. Yeo, S. Lim, I. H. Choe et al., "Acupuncture stimulation on GB34 activates neural responses associated with Parkinson's disease," CNS Neuroscience and Therapeutics, vol. 18, no. 9, pp. 781-790, 2012. 


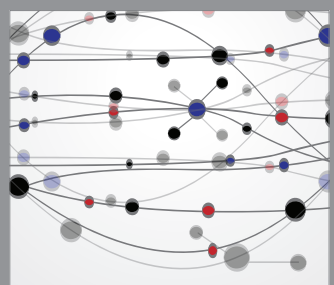

The Scientific World Journal
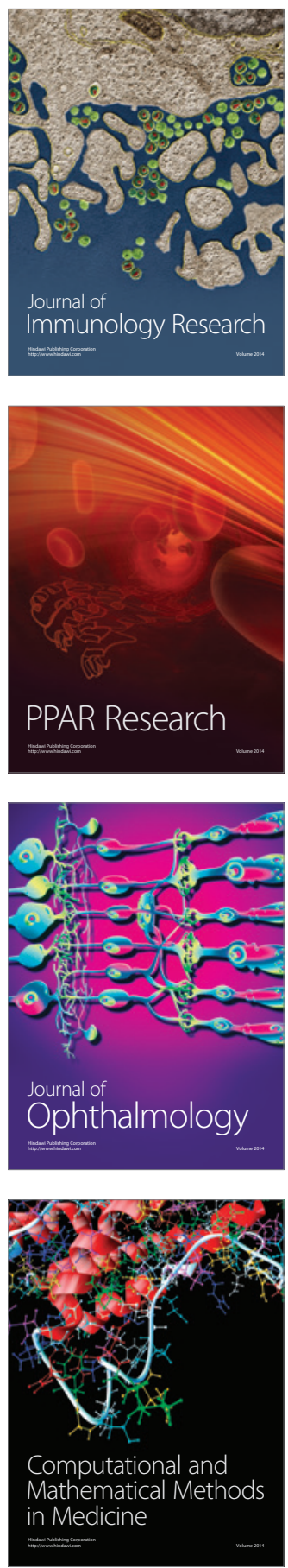

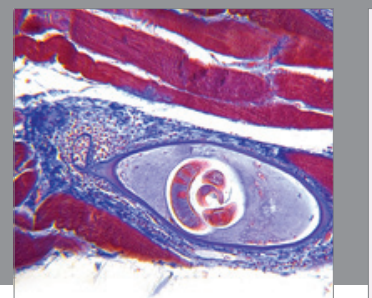

Gastroenterology

Research and Practice
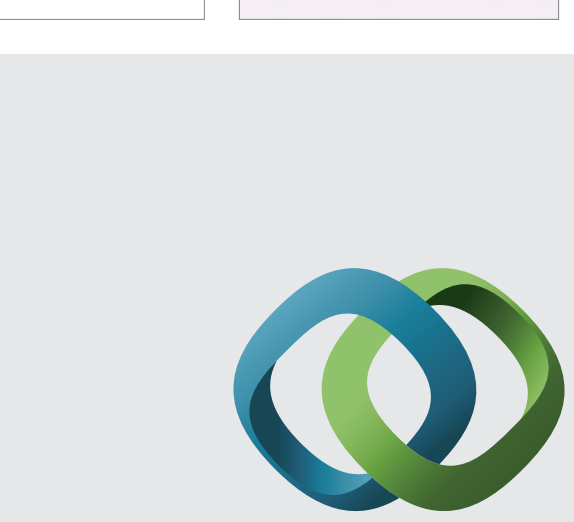

\section{Hindawi}

Submit your manuscripts at

http://www.hindawi.com
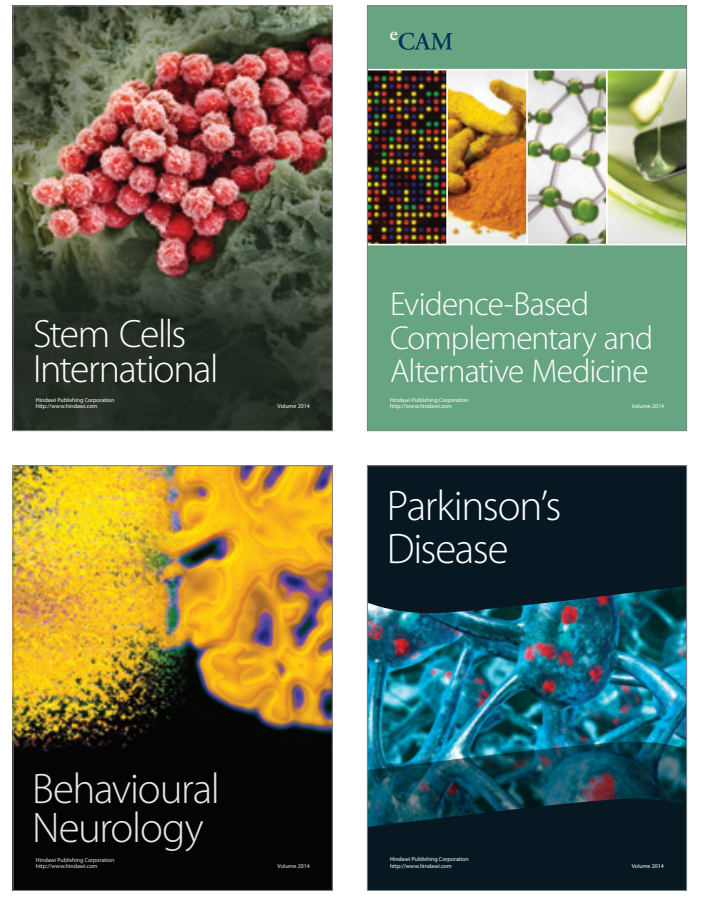
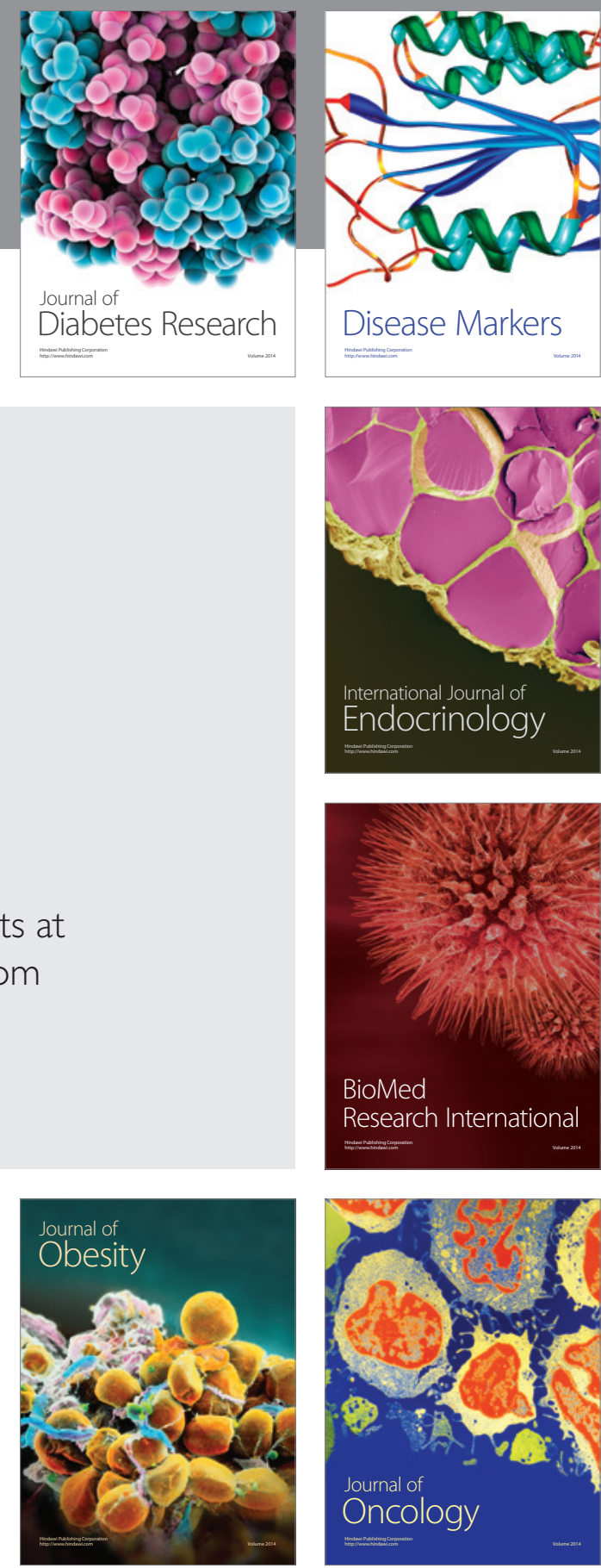

Disease Markers
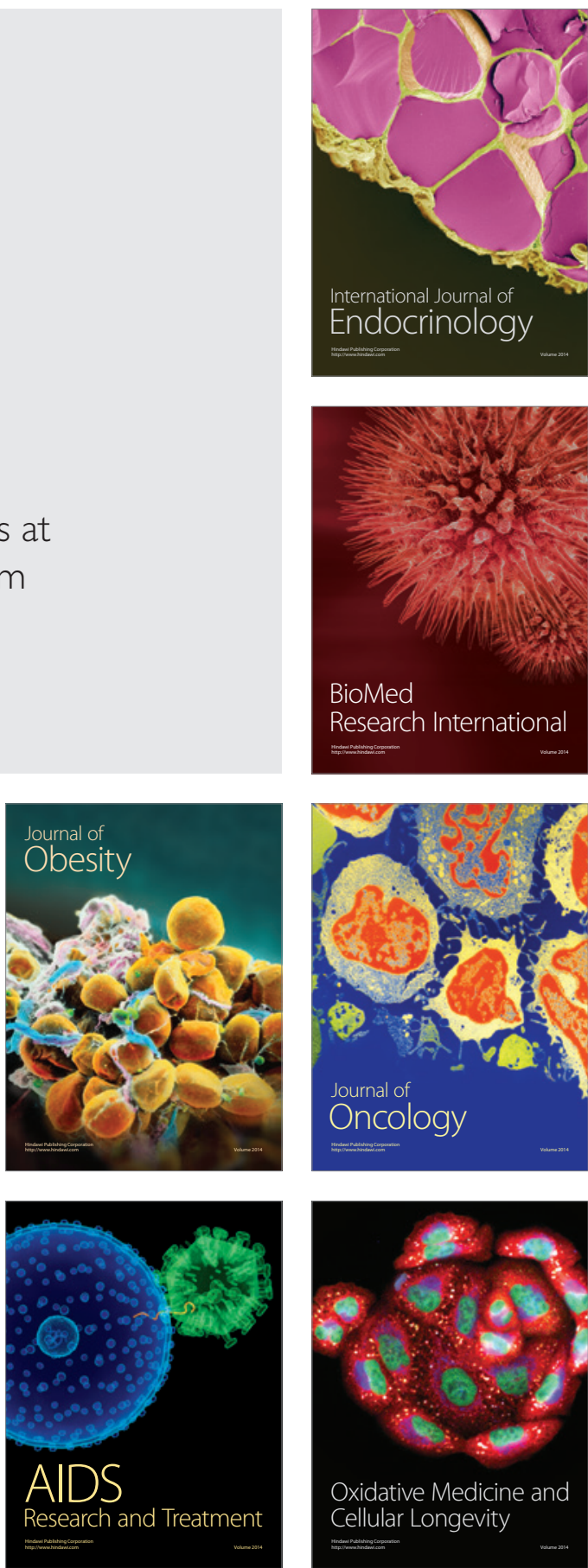